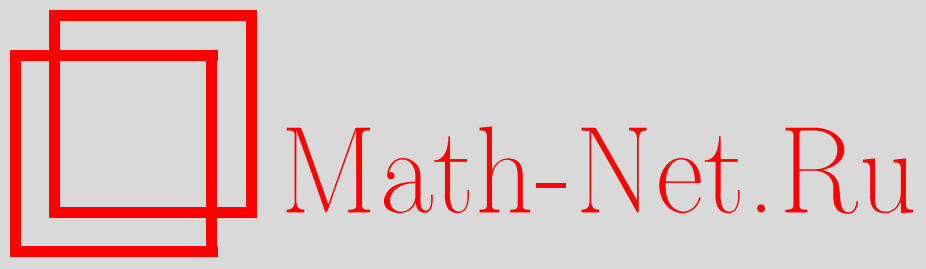

В. М. Мануйлов, Об асимптотических гомоморфизмах в алгебры Калкина, Функи. анализ и его прил., 2001, том 35, выпуск 2, 81-84

DOI: https://doi.org/10.4213/faa250

Использование Общероссийского математического портала MathNet.Ru подразумевает, что вы прочитали и согласны с пользовательским соглашением

http://www . mathnet.ru/rus/agreement

Параметры загрузки:

IP: 35.173 .137 .237

26 апреля 2023 г., 13:12:13

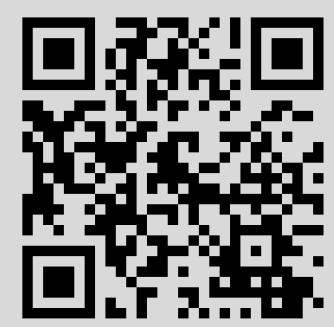


(1991). 6. Ilyashenko Yu., Yakovenko S. Amer. Math. Soc. Transl. Ser. 2, Vol. 165, Amer. Math. Soc., Providence, RI, 1995, pp. 1-20, 21-95. 7. Ilyashenko Yu., Kaloshin V. In: Proceedings of the Arnoldfest. Fields Inst. Comm., Vol. 24, Amer. Math. Soc, 1999, pp. 241271. 8. Хованский А. Г. Малочлены. Библиотека математика, вып. 2, Фазис, М., 1997. 9. Шильников Л. П. ДАН СССР, 160, 558-561 (1965).

Московский государственный университет

им. М. В. Ломоносова,

Поступило в редакцию механико-математический факультет

28 декабря 1998 г.

e-mail: kaloshin@math.princeton.edu

УДК 517.98

\title{
Об асимптотических гомоморфизмах в алгебры Калкина*
}

\author{
(c) 2001. В. М. МАНУЙЛОВ
}

Задача классификации расширений $C^{*}$-алгебр послужила стимулом проникновения топологических методов в теорию $C^{*}$-алгебр [3]. Как показано в [7], расширения ядерных $C^{*}$-алгебр описываются в терминах $K K$-функтора Каспарова, однако в общем случае пока подобного описания нет. В настоящей заметке мы определяем группу так называемых фантомных расширений (возможно, пустую) и в случае, когда $A$ является двукратной надстройкой, даем описание ее расширений в терминах $E$-теории Конна-Хигсона [5] и группы фантомных расширений.

Для $C^{*}$-алгебр $A$ и $B$ через $[[A, B]]$ мы обозначаем множество гомотопических классов асимптотических гомоморфизмов из $A$ в $B[5,6]$. Мы предполагаем, что $A$ сепарабельна, а $B$ имеет строго положительный элемент. Через $\operatorname{Ext}(A, B)$ мы обозначаем множество гомотопических классов гомоморфизмов из $A$ в алгебру Калкина $Q(B \otimes \mathscr{K})=M(B \otimes \mathscr{K}) / B \otimes \mathscr{K}$, где $\mathscr{K}$ есть $C^{*}$-алгебра компактных операторов, а $M$ обозначает алгебру мультипликаторов [13]. С помощью инварианта Басби [4] это множество отождествляется с множеством расширений алгебры $A$ посредством алгебры $B \otimes \mathscr{K}$. Аналогично, через $\operatorname{Ext}^{a s}(A, B)$ мы обозначаем множество гомотопических классов асимптотических гомоморфизмов алгебры $A$ в $Q(B \otimes \mathscr{K})$. (Асимптотические) гомоморфизмы $f_{0}, f_{1}: A \rightarrow Q(B \otimes \mathscr{K})$ называются гомотопными, если существует (асимптотический) гомоморфизм $F: A \rightarrow Q(B \otimes \mathscr{K} \otimes C[0,1])$, композиции которого с гомоморфизмами вычисления в 0 и 1 совпадают с $f_{0}$ и $f_{1}$ соответственно. Все указанные множества обладают естественной структурой абелевой группы в случае, когда $A$ является надстройкой, т.е. $A=S D=C_{0}(\mathbb{R}) \otimes D$ для некоторой $C^{*}$-алгебры $D$.

Так как любой настоящий гомоморфизм можно рассматривать как асимптотический, имеется естественное отображение

$$
i: \operatorname{Ext}(A, B) \rightarrow \operatorname{Ext}^{a s}(A, B) .
$$

Хотя типично асимптотических гомоморфизмов больше, чем настоящих, мы показываем, что отображение $i$ эпиморфно, если $A-$ надстройка. Заодно мы получим новое описание $E$-теории.

*Работа частично поддержана РФФИ (грант №99-01-01201). 
При работе с асимптотическими гомоморфизмами удобным инструментом является возможность их дискретизации, предложенная Мищенко $[12,9,10]$. Дискретным асимптотическим гомоморфизмом $\varphi=\left(\varphi_{n}\right)_{n \in \mathbb{N}}: A \rightarrow B \otimes \mathscr{K}$ мы называем асимптотический гомоморфизм, дополнительно удовлетворяющий условию $\lim _{n \rightarrow \infty}\left\|\varphi_{n+1}(a)-\varphi_{n}(a)\right\|=0$ для каждого $a \in A$. Без ограничения общности можно считать последовательность отображений $\varphi_{n}$ равномерно непрерывной [8], а благодаря теореме выбора [2] можно получить равномерно непрерывную последовательность поднятий $\varphi_{n}^{\prime}: A \rightarrow M(B \otimes \mathscr{K})$.

Для $a, b \in A, \lambda \in \mathbb{C}$ положим

$$
\begin{aligned}
P_{n}^{\prime}(a, b) & =\varphi_{n}^{\prime}(a) \varphi_{n}^{\prime}(b)-\varphi_{n}^{\prime}(a b), \\
L_{n}^{\prime}(a, b, \lambda) & =\varphi_{n}^{\prime}(a)+\lambda \varphi_{n}^{\prime}(b)-\varphi_{n}^{\prime}(a+\lambda b), \\
A_{n}^{\prime}(a) & =\varphi_{n}^{\prime}(a)^{*}-\varphi_{n}^{\prime}\left(a^{*}\right) .
\end{aligned}
$$

Мы отождествляем $B \otimes \mathscr{K}($ соответственно $M(B \otimes \mathscr{K}))$ с $C^{*}$-алгеброй компактных (соответственно допускающих сопряженный) операторов в стандартном гильбертовом $C^{*}$-модуле $B \otimes l_{2}(\mathbb{N})=l_{2}(B)$ и диагональность операторов понимаем в этом смысле. Ниже мы строим отображение $\widetilde{C H}: \operatorname{Ext}^{a s}(A, B) \rightarrow$ $[[S A, B \otimes \mathscr{K}]]$, являющееся ключевым в настоящей заметке и продолжающее аналогичное отображение $C H$ Конна-Хигсона [5]. Ясно, что это отображение достаточно определить для дискретных асимптотических гомоморфизмов. Для этого нужно более аккуратно, чем в конструкции Конна-Хигсона, выбрать квазицентральную аппроксимативную единицу [1].

Лемма 1. Пусть $\left(\varphi_{n}\right)_{n \in \mathbb{N}}: A \rightarrow Q(B \otimes \mathscr{K})-$ дискретный асимптотический гомоморфизм. Тогда существуют репараметризация последовательности $\left(\varphi_{n}\right)_{n \in \mathbb{N}}$ и аппроксимативная единица $\left(u_{n}\right)_{n \in \mathbb{N}} \subset B \otimes \mathscr{K}$, удовлетворяющие условиям

(i) $\lim _{n \rightarrow \infty}\left\|\left[\varphi_{n}^{\prime}(a), u_{n}\right]\right\|=0$ для каждого $a \in A$;

(ii) $\lim _{n \rightarrow \infty}\left\|\alpha\left(u_{n}\right) P_{n}^{\prime}(a, b)\right\|=\lim _{n \rightarrow \infty}\left\|\alpha\left(u_{n}\right) L_{n}^{\prime}(a, b, \lambda)\right\|=\lim _{n \rightarrow \infty}\left\|\alpha\left(u_{n}\right) A_{n}^{\prime}(a)\right\|=0$ для всех $\alpha \in C_{0}(0,1), a, b \in A, \lambda \in \mathbb{C}$;

(iii) $\lim _{n \rightarrow \infty}\left\|u_{n+1}-u_{n}\right\|=0$;

(iv) каждьй оператор $u_{n}$ является диагональным, $u_{n}=\operatorname{diag}\left\{u_{n}^{1}, u_{n}^{2}, \ldots\right\}, c$ диагональными әлементами $u_{n}^{i} \in B, u \lim _{i \rightarrow \infty} \sup _{n}\left\|u_{n}^{i+1}-u_{n}^{i}\right\|=0$.

Положим

$$
\widetilde{C H}(\varphi)_{n}(\alpha \otimes a)=\alpha\left(u_{n}\right) \varphi_{n}^{\prime}(a), \quad \alpha \in C_{0}(0,1), a \in A,
$$

где $\left(u_{n}\right)_{n \in \mathbb{N}}$ удовлетворяет условиям леммы 1 . Пункты (i)-(iii) леммы 1 гарантируют, что $\left(\widetilde{C H}(\varphi)_{n}\right)_{n \in \mathbb{N}}-$ дискретный асимптотический гомоморфизм из $S A$ в $B \otimes \mathscr{K}$. Легко проверить, что отображение $\widetilde{C H}$ определено корректно и что коммутативна диаграмма

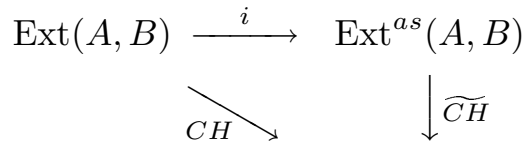

$[[S A, B \otimes \mathscr{K}]]$ 
Определим гомоморфизм $g: C_{0}(0,1) \rightarrow Q(\mathscr{K})$ формулой $g\left(\alpha_{0}\right)=q(T)-1$ по модулю компактных операторов, где $\alpha_{0}=e^{2 \pi i x}-1-$ образующая в $C_{0}(0,1)$, а $T$ - правый сдвиг в гильбертовом пространстве $l_{2}(\mathbb{N})$. Пусть $\iota: Q(\mathscr{K}) \otimes B \otimes \mathscr{K} \subset$ $Q(B \otimes \mathscr{K} \otimes \mathscr{K}) \cong Q(B \otimes \mathscr{K})-$ стандартное вложение и

$$
j=\iota \circ\left(g \otimes \operatorname{id}_{B \otimes \mathscr{K}}\right): S B \otimes \mathscr{K} \rightarrow Q(\mathscr{K}) \otimes B \otimes \mathscr{K} \rightarrow Q(B \otimes \mathscr{K}) .
$$

Гомоморфизм $j$ индуцирует отображение $j_{*}:[[A, S B \otimes \mathscr{K}]] \rightarrow \operatorname{Ext}^{a s}(A, B)$. Если $S:[[A, B \otimes \mathscr{K}]] \rightarrow[[S A, S B \otimes \mathscr{K}]]-$ отображение надстройки, то композиция $M=j_{*} \circ S$ задает отображение

$$
M:[[A, B \otimes \mathscr{K}]] \rightarrow \operatorname{Ext}^{a s}(S A, B) .
$$

Пусть

$\operatorname{Bott}_{1}: \operatorname{Ext}^{a s}(A, B) \rightarrow \operatorname{Ext}^{a s}\left(S^{2} A, B\right) \quad$ и $\quad \operatorname{Bott}_{2}:[[A, B \otimes \mathscr{K}]] \rightarrow\left[\left[S^{2} A, B \otimes \mathscr{K}\right]\right]$ - гомоморфизмы Ботта.

TEOPEMA 2. $M \circ \widetilde{C H}=$ Bott $_{1} u \widetilde{C H} \circ M=$ Bott $_{2}$.

Второе равенство доказывается непосредственной проверкой, а доказательство гомотопности асимптотических изоморфизмов $\operatorname{Bott}_{1}(\varphi)$ и $M \circ \widetilde{C H}(\varphi)$, где $\varphi=\left(\varphi_{n}\right)_{n \in \mathbb{N}}: A \rightarrow Q(B \otimes \mathscr{K})$, можно заменить на построение гомотопий, соединяющих каждый из этих двух асимптотических гомоморфизмов с настоящим гомоморфизмом $f: S^{2} A \rightarrow Q(B \otimes \mathscr{K})$, определенным формулой

$$
f\left(\alpha \otimes\left(e^{2 \pi i x}-1\right) \otimes a\right)=\left(\bigoplus_{n=1}^{\infty} \alpha\left(u_{n}\right) \varphi_{n}^{\prime}(a)\right)(T-1),
$$

где $\alpha, e^{2 \pi i x}-1 \in C_{0}(0,1), a \in A$ и $T-$ правый сдвиг в гильбертовом модуле $l_{2}(B)$.

Поскольку гомоморфизмы Ботта являются изоморфизмами в случае, когда $A$ - надстройка, отображения $\widetilde{C H}$ и $M$ взаимно обратны.

Следствие 3. Если $A-$ надстройка, то отображение $\widetilde{C H}: \operatorname{Ext}^{a s}(A, B) \rightarrow$ $[[S A, B \otimes \mathscr{K}]]$ является изоморфизмом.

Назовем гомоморфизм $f: A \rightarrow Q(B \otimes \mathscr{K})$, который гомотопически тривиален в классе асимптотических гомоморфизмов, фантомным, и обозначим через $\operatorname{Ext}^{p h}(A, B)$ множество гомотопических классов фантомных гомоморфизмов.

СледствиЕ 4. Если А является двукратной надстройкой, то имеет место естественное разложение в прямую сумму $\operatorname{Ext}(A, B)=\operatorname{Ext}^{p h}(A, B) \oplus$ $\operatorname{Ext}^{a s}(A, B)$.

ДокАЗАТЕЛЬСтво. Рассмотрим последовательность

$$
0 \longrightarrow \operatorname{Ext}^{p h}\left(S^{2} A, B\right) \longrightarrow \operatorname{Ext}\left(S^{2} A, B\right) \stackrel{C H}{\longrightarrow} \operatorname{Ext}^{a s}\left(S^{2} A, B\right) \longrightarrow 0 .
$$

В [11] показано, что гомоморфизм $C H$ сюръективен; значит, последовательность $(2)$ точна. В $[9,10,11]$ построено естественное отображение $E:[[A, B \otimes \mathscr{K}]] \rightarrow$ $\operatorname{Ext}(S A, B)$ и доказано, что композиция $C H \circ E$ является изоморфизмом в случае, когда $A-$ надстройка. После отождествления $\operatorname{Ext}^{a s}\left(S^{2} A, B\right) \cong[[S A, B \otimes \mathscr{K}]]$ ясно, что гомоморфизм $E$ служит расщеплением точной последовательности (2).

ЗАМЕчАНИЕ 5. Если $A$ одновременно является ядерной $C^{*}$-алгеброй и надстройкой, то группы $\operatorname{Ext}(A, B)$ и $[[A, B \otimes \mathscr{K}]]$ совпадают и $\operatorname{Ext}^{p h}(A, B)=0$. 


\title{
ЛИТЕРАТУРА
}

1. Arveson $W$. Duke Math. J., 44, 329-355 (1977). 2. Bartle R. G., Graves L. M. Trans. Amer. Math. Soc., 72, 400-413 (1952). 3. Brown L. G., Douglas R. G., Fillmore P. A. Ann. Math. (2), 105, 265-324 (1977). 4. Busby R. Trans. Amer. Math. Soc., 132, 79-99 (1968). 5. Connes A., Higson N. C. R. Acad. Sci. Paris, Sér. I, 311, 101-106 (1990). 6. Dădărlat M., Loring T. A. J. Funct. Anal., 126, 367-383 (1994). 7. Каспаров Г. Г. Изв. AН СССР, сер. матем., 44, 571-636 (1980). 8. Loring T. A. In: Operator Algebras and Quantum Field Theory (Rome, 1996), Internat. Press, 1997, pp. 111-122. 9. Мануйлов В. М., Мищенко А. C. Матем. сб., 189, № 10, 53-72 (1998). 10. Manuilov V. M., Thomsen $K$. Adv. Math., 154, 258-279 (2000). 11. Мануйлов B. М., Томсен К. Алгебра и анализ, 12, вып. 5, 142-157 (2000). 12. Mishchenko A. S., Mohammad N. Asymptotic representations of discrete groups. In: Lie Groups and Lie Algebras. Their Representations, Generalizations and Applications, Mathematics and its Applications, Vol. 433. Kluwer Acad. Publ., Dordrecht, 1998, pp. 299-312. 13. Pedersen G. K. $C^{*}$-algebras and their automorphism groups. Acad. Press, London-New York-San Francisco, 1979.

Московский государственный университет, механико-математический факультет e-mail: manuilov@mech.math.msu.su

Поступило в редакцию 13 марта 2000 г.

УДК 517.9

\section{О рангах эргодического автоморфизма $T \times T$}

\author{
(c) 2001. В. В. Рыжиков
}

1. С автоморфизмом $S$ пространства Лебега $(X, \mu), \mu(X)=1$, связан числовой инвариант $\beta(S)$, называемый локальным рангом. Значение $\beta(S)$ определяется как максимальное число $\beta \geqslant 0$, такое, что найдется последовательность $\xi_{j}$ измеримых конечных разбиений вида $\xi_{j}=\left\{B_{j}, S B_{j}, S^{2} B_{j}, \ldots, S^{h_{j}-1} B_{j}, A_{j}^{1}, A_{j}^{2}, \ldots\right.$, $\left.A_{j}^{n(j)}\right\}$ (здесь $B_{j}$ и $A_{j}^{1}, A_{j}^{2}, \ldots, A_{j}^{n(j)}-$ некоторые измеримые подмножества пространства $X$ ), которая стремится к разбиению на точки (мы используем обозначение $\xi_{j} \rightarrow \varepsilon$ ), причем $\mu\left(U_{j}\right) \rightarrow \beta$, где $U_{j}=\bigsqcup_{0 \leqslant k<h_{j}} S^{k} B_{j}$ (множества $U_{j}$ будем называть $\beta$-башнями).

Говорят, что автоморфизм $S$ имеет конечный ранг, если для некоторого натурального $r$ найдется последовательность разбиений $\eta_{j} \rightarrow \varepsilon$ вида

$$
\eta_{j}=\left\{B_{j}^{1}, S B_{j}^{1}, S^{2} B_{j}^{1}, \ldots, S^{h_{j}^{1}} B_{j}^{1}, \ldots, B_{j}^{r}, S B_{j}^{r}, S^{2} B_{j}^{r}, \ldots, S^{h_{j}^{r}} B_{j}^{r}, Y_{j}\right\},
$$

где $h_{j}^{1}, \ldots, h_{j}^{r} \rightarrow \infty$ и $\mu\left(Y_{j}\right) \rightarrow 0$. Минимальное такое число $r$, если оно существует, обозначается через $\operatorname{Rank}(S)$ и называется рангом автоморфизма $S$. Если автоморфизм не обладает конечным рангом, полагаем $\operatorname{Rank}(S)=\infty$.

Автоморфизмам конечного и положительного локального ранга посвящен обзор [2]. Пример автоморфизма $S$ с $\beta(S)>0$ и $\operatorname{Rank}(S)=\infty$ впервые построил С. Ференци [2].

2. В неопубликованных лекциях [4] А. Каток рассмотрел слабо перемешивающие автоморфизмы $T$, такие, что локальный ранг автоморфизма $T \times T$ был не меньше, чем $1 / 4$ (обсуждение этих примеров см. в [3]). Мы покажем, что для 